\title{
Comparison of mouse models reveals a molecular distinction between psychotic illness in PWS and schizophrenia
}

\author{
Simona K. Zahova ${ }^{1}$, Trevor Humby ${ }^{2}$, Jennifer R. Davies ${ }^{1}$, Joanne E. Morgan ${ }^{1}$ and Anthony R. Isles (iD) ${ }^{1 凶}$
}

(c) The Author(s) 2021

Prader-Willi Syndrome (PWS) is a neurodevelopmental disorder caused by mutations affecting paternal chromosome 15q11-q13, and characterized by hypotonia, hyperphagia, impaired cognition, and behavioural problems. Psychotic illness is a challenging problem for individuals with PWS and has different rates of prevalence in distinct PWS genotypes. Previously, we demonstrated behavioural and cognitive endophenotypes of relevance to psychiatric illness in a mouse model for one of the associated PWS genotypes, namely PWS-IC, in which deletion of the imprinting centre leads to loss of paternally imprinted gene expression and over-expression of Ube3a. Here we examine the broader gene expression changes that are specific to the psychiatric endophenotypes seen in this model. To do this we compared the brain transcriptomic profile of the PWS-IC mouse to the PWS-cr model that carries a deletion of the PWS minimal critical interval spanning the snoRNA Snord116 and Ipw. Firstly, we examined the same behavioural and cognitive endophenotypes of relevance to psychiatric illness in the PWS-cr mice. Unlike the PWS-IC mice, PWS-cr exhibit no differences in locomotor activity, sensory-motor gating, and attention. RNA-seq analysis of neonatal whole brain tissue revealed a greater number of transcriptional changes between PWS-IC and wild-type littermates than between PWS-cr and wild-type littermates. Moreover, the differentially expressed genes in the PWS-IC brain were enriched for GWAS variants of episodes of psychotic illness but, interestingly, not schizophrenia. These data illustrate the molecular pathways that may underpin psychotic illness in PWS and have implications for potential therapeutic interventions.

Translational Psychiatry (2021)11:433; https://doi.org/10.1038/s41398-021-01561-x

\section{INTRODUCTION}

Prader-Willi syndrome (PWS) is a neurodevelopmental disorder primarily caused by mutations affecting paternally expressed genes (PEGs) on the imprinted $15 q 11.2-q 13$ region. This interval includes several genes expressed only from the paternal chromosome (MKRN3, MAGEL2, NECDIN, NPAP1, SNURF-SNRPN, cluster of non-coding RNAs), as well as two genes expressed only from the maternal chromosome (UBE3A, ATP10) [1]. The disruption of this locus is associated with hypotonia, growth retardation, hyperphagia, mild to a moderate learning disability, as well as a range of psychiatric conditions [2, 3]. Upwards of $20 \%$ of PWS patients are formally diagnosed with major depressive disorder (MDD), while $50-70 \%$ score highly on questionnaires for anxious and depressive episodes [4]. Phenotypes of reduced attention and poor impulse control are also highly prevalent and are reported to be intensified in children with PWS compared to their peers with learning disabilities only $[5,6]$. Furthermore, between $16 \%$ and $32 \%$ of patients experience episodes of psychosis, including delusional ideation and hallucinations $[4,5,7,8]$.

The prevalence and variation of psychiatric endophenotypes in PWS are influenced by individual genotypes. Individuals with PWS can be roughly divided into two groups based on distinct genotypes. The first and most prevalent group ( 75\%) consists of those that carry a paternally derived deletion spanning the genes of the 15q11-q13 (delPWS) region. In recent years, several case studies have reported paternally derived microdeletions of the 15q11.2-q13 non-coding RNAs in individuals that exhibit growth retardation, hyperphagia, and hypotonia [9-11]. Since these are regarded as the core features of PWS, the region covered by the microdeletions, in which non-coding PEGs SNORD116 and IPW are deleted, is referred to as the PWS critical interval (PWS-cr).

The second most common PWS genotype is maternal uniparental disomy of chromosome 15 (mUPD15), found in about $25 \%$ of PWS patients. As well as the core phenotypes, population studies of individuals with PWS report increased incidence and severity of psychosis in those with mUPD15 compared to delPWS, including a higher rate of diagnoses of schizoaffective and bipolar disorders [12,13]. mUPD15 leads to loss of expression of all PEGs, including those in the PWS-cr, and also to an overexpression of the two maternally expressed genes (MEGs) in the 15q11-q13 imprinted interval [1]. A similar molecular profile (loss of PEGs, overexpression of MEGs) is seen in the rare imprinting centre (IC) mutation genotype $(<5 \%)$ [14], which also shows the incidence of psychotic illness [15]. These findings have led to the suggestion that the overexpression of MEGs, and specifically UBE3A, might be the main contributors to psychiatric illness in PWS [1], an idea supported by the increased incidence of psychotic illness in individuals carrying maternally-derived copy number variation (CNV) duplications at 15q11-q13 [16]. Nevertheless, delPWS patients can also exhibit depression, anxiety and, albeit with

\footnotetext{
${ }^{1}$ MRC Centre for Neuropsychiatric Genetics and Genomics, School of Medicine, Cardiff University, Cardiff, UK. ${ }^{2}$ School of Psychology, Cardiff University, Cardiff, UK. email: IslesAR1@cardiff.ac.uk
} 
reduced incidence and severity, psychotic episodes [7, 17], and recent genomic studies linked variation in the PWS PEGs MAGEL2, NECDIN, and SNORD116, to scoring highly on the Schizotypal Personality Questionnaire $[18,19]$. Taken together, this suggests a potential combinatorial role for both the PWS PEGs and MEGs in psychiatric illness. However, as yet the mechanism leading to psychiatric illness remains unclear.

To address this, we have used mouse models to investigate behavioural and cognitive phenotypes of relevance to psychiatric illness seen in PWS. Previously, we have examined behavioural and cognitive phenotypes in a PWS imprinting centre deletion (PWS-IC) mouse, which models the rare IC mutation genotype and demonstrates loss of expression of PEGs and overexpression of MEGs $[14,20]$, matching that observed in individuals with mUPD15. We have shown that the PWS-IC model recapitulates many of the behavioural and cognitive deficits of relevance to PWS, including hypoactivity, abnormal sensory-motor gating, reduced attention, and greater impulsivity [20-22]. Here, we examine the behaviour of another PWS mouse model carrying a deletion of PWS-Cr, in order to investigate whether the PWS-cr contributes to the endophenotypes of relevance to psychiatric illness and cognition observed in the PWSIC mouse model. Both models show the growth [20, 23, 24], endocrine [23, 25], and hyperphagia [26-28] phenotypes central to PWS. We tested whether hypoactivity, abnormal sensory-motor gating, reduced attention, and greater impulsivity are indeed limited to the PWS-IC mice by conducting the same behavioural characterisation in PWS-cr mice. Furthermore, we attempted to examine the broader molecular changes that may underpin these cognitive and psychiatric endophenotypes specifically by using RNA-seq to examine gene expression changes in the brain of both PWS models. The behavioural differences between models were reflected in distinct profiles of differentially expressed genes (DEGs) and isoform changes between PWS-IC and PWS-cr brain. Critically, the DEGs and isoform changes in PWS-IC, but not PWS-cr, were enriched for genetic variants associated with psychotic illness, but interestingly not schizophrenia. Our findings shed light on a molecular basis of behavioural and cognitive problems seen in PWS and may have implications for the treatment of psychotic illness in individuals with genetic lesions at $15 q 11-q 13$.

\section{MATERIALS AND METHODS \\ Behavioural experiments}

Animal husbandry. Due to the genomic imprinting of the 15q11.2-q13 locus, heterozygous mice carrying the deletion on the paternally inherited chromosome are expected to have a complete loss of expression of the critical interval. PWS-cr ${ }^{\mathrm{m}+/ \mathrm{p}-}$ mice (B6(Cg)-Snord116 $\left.6^{\text {tm } 1 \text { Uta }} / \mathrm{J}\right)$ were purchased from The Jackson Laboratory. The females were mated with wildtype $\mathrm{C} 57 \mathrm{BL} / 6$ males in order to generate PWS-cr ${ }^{\mathrm{m}-/ \mathrm{p}+}$ mice, which carry the deletion on the silenced maternal copy of the critical interval and are therefore expected not to exhibit any of the phenotypes associated with it. The behavioural cohort of 24 PWS- $\mathrm{Cr}^{\mathrm{m}+/ \mathrm{p}-}$ ( $16 \mathrm{female}, 8$ male) and 31 wild types (17 female, 14 male) was produced by breeding PWS-cr ${ }^{\mathrm{m}-/ \mathrm{p}+}$ males with wild type CD1 females in order to exactly replicate the genetic background that we have used in our previous study examining the behaviour of a PWS-IC mouse model [20]. Weaning took place at approximately four weeks of age, when animals were housed in singlesex and mixed genotype cages of 2-4 individuals. Ear tissue was collected for genotyping and identification. Experimental testing began at approximately 8-9 weeks of age and took place in the order in which the tests are described below. The number of animals used was determined on the basis of data variability in previous experiments and our ability to determine differences by genotype or sex, and interaction between genotype and sex. Due to the need to balance genotypes within cages and during testing, the experimenter was not blinded.

The animals were handled daily for two weeks prior to the start of experimentation in order to adapt them to be picked up. All mice were kept at a $12 \mathrm{~h}$ light/dark cycle, with the lights being switched on at 7:00 h every morning. Access to water and food was ad libitum, until two weeks before the start of the reward preference test, when the animals were gradually introduced to a water deprivation regime. During the first two days of this regime, the animals were given access to water for $4 \mathrm{~h}$ per day. After that, the access to water was limited to $2 \mathrm{~h}$ per day. The water deprivation regime continued all through the reward preference test and the 5-choice serial reaction time task. Water bottles were provided in the home-cages immediately after experimentation.

All procedures were conducted in accordance with the requirements of the UK Animals (Scientific Procedures) Act 1986, under the remit of Home office license number 30/3375 with ethical approval at Cardiff University.

Elevated plus maze and open field. The elevated plus maze (EPM) and open field test (OF) were used to measure anxiety-related behaviours $[29,30]$. The EPM consisted of a plus-shaped platform, with two enclosed arms $(19 \times 8 \times 15 \mathrm{~cm})$ and two open, exposed arms $(19 \times 8 \mathrm{~cm})$. The maze was lifted $50 \mathrm{~cm}$ above the ground. The animals were placed in one of the enclosed arms of the maze at the beginning of the task, and allowed to freely explore for $5 \mathrm{~min}$. The OF apparatus consisted of a square arena $(75 \times 75 \mathrm{~cm})$ enclosed by walls $(45 \mathrm{~cm})$. The surface of the arena was divided into the central zone $(45 \times 45 \mathrm{~cm})$ and the outer zone. The animals were placed into the outer zone of the arena and allowed to freely explore for $10 \mathrm{~min}$. The movement of the animals in the EPM and OF was detected by a camera attached to a computer with Ethovision software (XT, Noldus, Netherlands). Time spent in movement during the trials was calculated post-hoc by dividing the distance by the velocity.

Locomotor activity. Spontaneous locomotor activity (LMA) of mice was explicitly measured in custom-made chambers $(21 \times 36 \times 20 \mathrm{~cm})$ fitted with infrared beams, situated $3 \mathrm{~cm}$ from either end of the box and $1 \mathrm{~cm}$ from the floor of the box. The test was run in complete darkness in order to remove the anxiogenic effect of light. The mice were placed in the boxes and allowed to roam freely for $2 \mathrm{~h}$, while the disturbance of the beams by their movement was recorded by an Acorn computer with an Arachnid software (Cambridge Cognition LTD). The number of beam breaks and the consecutive breaking of the two infrared beams (referred to as a "run") were considered the main measures of locomotor activity. The animals were assessed at the same time for two consecutive days, in order to assess habituation to a novel environment within and between sessions.

Acoustic startle and pre-pulse inhibition. Acoustic startle response (ASR) and pre-pulse inhibition (PPI) were used to test for phenotypes associated with psychotic and affective illness [31]. ASR and PPI were measured in a soundproofed SR LAB startle chamber with a speaker (San Diego Instruments), in which the mice were securely placed inside a plexiglass tube ( $3.5 \mathrm{~cm} \mathrm{dia)}$. The trial commenced with $5 \mathrm{~min}$ of $70 \mathrm{~dB}$ white noise in order to allow for habituation to the environment. After habituation, the mice were exposed to a range of acoustic stimuli, including pulse-alone stimuli at $120 \mathrm{~dB}$ and $105 \mathrm{~dB}$ above background noise $(70 \mathrm{~dB})$ for $40 \mathrm{~ms}$, then startle stimuli preceded by $20 \mathrm{~ms}$ pre-pulses of $8 \mathrm{~dB}$ and $16 \mathrm{~dB}$, $100 \mathrm{~ms}$ prior to startle stimuli. All startle and pre-pulse stimuli were presented against a background noise of $70 \mathrm{~dB}$. The startle movement in response to the noise was recorded with a piezoelectric accelerometer and normalized by body weight before analysis. PPI of the ASR was calculated as the percentage reduction in startle amplitude between responses to startling stimuli alone (averaged cross 13 trials) and pre-pulse trials (averaged for the 5 presentations at each pre-pulse amplitude.

Reward preference test. Prior to the 5-CSRT and after a transition to restricted home-cage water access, animals were gradually exposed to the substance ( $10 \%$ condensed milk in tap water) which was to be used as a reward for the tasks later on. The animals were placed individually in a cage without sawdust, for a 10 min session each day, over a 7-day period. Each cage contained two small plastic containers $(\sim 1 \mathrm{~cm}$ high $\times \sim 2 \mathrm{~cm}$ diameter) secured to the floor by Velcro, placed equidistant from the end wall, and evenly spaced across the width of the cage. On the first two days, both containers contained tap water. On the following four days, one container contained the condensed milk dilution and the other - water, with the positions alternating each day of testing. On the final day, both containers were filled with the condensed milk dilution. All consumption was measured in grams by weighing the containers before and after the task. The preference for the reward was calculated for days 3-6 as a percentage of the total volume consumed.

5-choice serial reaction time task. The 5-choice serial reaction time task (5CSRTT) is commonly used to measure visuo-spatial attention and 
impulsivity in rodent models of disease $[32,33]$. The 5 -CSRTT was commenced on the day following the final day of the reward preference test and broadly followed the protocol described by [34]. For this task, mice were trained to respond to a brief visual stimulus appearing pseudorandomly at one of five locations, in order to gain a condensed milk reward. The variables analysed as indicative of performance were accuracy (ratio of correct to total trials), omissions (ratio of omitted trials to total trials), and premature responses (number of responses that took place before the stimulus was presented). Other variables such as completed trials (calculated as ratio out of 60), correct reaction time, reward collection latency, number of food magazine entries, and the total number of trial perseveration were also kept track of for further analysis. Subjects were trained to a set baseline performance observed at a stimulus duration of $0.8 \mathrm{~s}$ ( $>30$ completed trials, $>75 \%$ accuracy, and $<25 \%$ omissions). Full details can be found in Supplementary information.

The conditions of the experiment were manipulated in order to study various aspects of attention and impulsivity. One of the manipulations entailed shortening the stimulus duration (SSD). During this session, stimuli of $0.6,0.4$, and $0.2 \mathrm{~s}$ were interspersed in pseudorandom order along with the baseline stimulus duration of $0.8 \mathrm{~s}$, in order to increase the difficulty of the task and to affect the accuracy of performance. In another manipulation lengthened it is (LITI) of $7 \mathrm{~s}, 9 \mathrm{~s}$, and $11 \mathrm{~s}$ were interspersed in pseudorandom order along with the baseline inter-trial interval of $5 \mathrm{~s}$, in order to induce premature responses and test for impulsive behaviour.

Data analysis. All data were analysed using R Studio 1.1.383 (R Studio, Inc). Linear models $(\mathrm{Im})$ and generalized linear models $(\mathrm{g} / \mathrm{m})$ were built with genotype and sex as fixed effects. Since interactions between sex and genotype were not observed, the effect of sex is not reported in this paper. However, the data, which are freely available on the 'Open science framework' (https://osf.io/wnx8r/) are identifiable and therefore separable by sex. Logistic glm from the binomial family was used to analyse all proportional data. Mixed Ims and glms with mouse ID as a random effect were built using the Ime4 package [35], for data in which multiple measures were taken from each individual at different conditions. In the LMA mixed model, day of testing and time bins were added as fixed effects. In the ASR mixed model, the startle trial number was added as a fixed effect. In the PPI mixed model, the different pre-pulse decibels ( $8 \mathrm{~dB}$, $16 \mathrm{~dB}$ ) were added as fixed effects. For the 5-CSRTT SSD and LITI manipulations, stimulus duration and inter-trial interval were also added as fixed effects. The $p$-values and chi-square values $\left(x^{2}\right)$ presented in the results were calculated using likelihood ratio tests run with the inbuilt ANOVA function of $R$ and comparing the full statistical models to reduced models with genotype removed as a fixed factor.

\section{RNA sequencing}

Tissue collection, preparation, and RNA sequencing. For the RNA sequencing experiments, whole-brain neonatal tissue was taken from 6 PWS-cr mice and 6 of their wild-type littermates (from 4 separate litters), and from 4 PWS-IC ${ }^{\mathrm{m+/p}-}$ mice and 6 of their wild type littermates (from 2 separate litters). The tissue was collected first thing in the morning (9-10:00 am) following birth and was snap-frozen on dry ice and stored at $-80^{\circ} \mathrm{C}$ until. RNA was extracted using the Direct-zol RNA Miniprep kit. Libraries were prepared with the KAPA mRNA HyperPrep kit (Roche Sequencing solutions) from total RNA of RQN $>8.5$ as assessed by the Fragment Analyser system (Agilent Technologies). $800 \mathrm{ng}$ of polyA selected RNA was used for each sample for a paired and unstranded sequencing approach. The target read depth was 80 million reads per sample. The mRNA was fragmented at $94^{\circ} \mathrm{C}$ for $6 \mathrm{~min}$ to achieve a mean insert size of 200-300 bp. A 8 cycles were used in the final amplification. A final bead clean-up step was added at the end of the protocol before the libraries were taken forward for NGS on an Illumina HiSeq4000 system.

RNA sequencing analysis. For analysis of differential gene expression, sequencing reads were mapped to the mouse genome (version $\mathrm{mm} 10$ ) using the STAR software package [36]. Unmapped reads were output into the BAM file with the Within KeepPairs option, which records unmapped mates for each alignment and keeps any unsorted output adjacent to its mapped mate. Multimapping alignment was done with a random order. The quantification of annotations was calculated with the GeneCounts option which outputs count reads per gene. The featureCounts software [37] was used to count the raw reads, which were subsequently analysed in R Studio 1.1.383 using the DESeq2 package [38], with genotype and litter as fixed effects into the statistical model. Hierarchical clustering was performed with the heatmap.2 package in $\mathrm{R}$, which uses Euclidean distances to calculate the difference between data points. The clustering showed an outlier in both PWS-Cr and PWS-IC samples, which were removed from further analysis. The UCSC genome browser was used for the visualization of the data. Differential isoform usage was pseudo-aligned to the mouse transcriptome ( $\mathrm{mm} 10)$ with the Kallisto software [39] which was also used to quantify the raw reads. The Kallisto files were then analysed in R studio via DEXSeq with the IsoformSwitchAnalyzeR package, which measures isoform usage by quantifying the fraction of isoform expression from the parent gene expression. The difference in usage between isoforms is then used to measure the effect size [40-42]. Prediction of protein domains was run on EBI's Pfam webserver, which performs bio sequence analysis using hidden Markov models [43]. The Pfam data were then integrated back into IsoformSwitchAnalyzer for final statistical analysis. Gender and litter were added as covariates into the statistical model. All $p$-values were adjusted using the Benjamini-Hochberg method. The data from the PWS-cr and PWS-IC models were analysed separately since the difference between the wild-type littermates of the two groups did not allow for pooling of the data. Genes that were differentially expressed or exhibited differential usage of isoforms between genotypes $\left(p_{\text {adj }}<0.05\right)$ were pooled together for functional enrichment analysis using the g: Profiler web server [44].

Common variant enrichment analysis. Genes that were differentially expressed or exhibited differential usage of isotopes between genotypes $\left(p_{\text {adj }}<0.05\right)$ were pooled together for common variant enrichment analysis. The mouse gene IDs were converted to their homologous human gene IDs. The genes were run for gene set analysis through MAGMA along with GWAS summary statistics for schizophrenia, psychosis, and chronic kidney disease [45-47]. Gene locations build 37 and reference data from the European population was downloaded from CNCR CTGLab (https://ctg. cncr.nl/software/magma).

\section{RESULTS}

\section{Elevated plus maze and open field test}

As expected, on the EPM all mice spent significantly more time of the 5 min session in the closed arms of the maze compared to the more anxiogenic open arms of the maze $\left(X^{2}(1)=138.98, p<0.001\right)$, but no effect of genotype on the percentage of time spent in the open arms of the $\operatorname{EPM}\left(F_{(3,50)}=0.132 ; p=0.361\right)$ (Fig. 1A), or on distance travelled through the area of the maze $\left(F_{(3,50)}=2.352, p=0.174\right)$. The number of head dips and stretch attends were also recorded as a measure of explorative behaviour through the EPM and showed no significant differences between PWS-cr mice and their wild type littermates $\left(F_{(3,50)}=0.7832, p=0.989 ; F_{(3,50)}=1.933, p=0.151\right)$.

During the OF test, mice spent significantly more time of the 10 min session in the outer areas of the arena than in the more anxiogenic centre square $\left.x^{2}(1)=452.2, p<0.001\right)$, but there was no effect of genotype on the percentage of time spent in the centre square $\left(F_{(3,50)}=0.084 ; p=0.361\right)$ (Fig. 1B) or on distance travelled through the arena $\left(F_{(3,50)}=4.55 ; p=0.472\right)$. Overall, the data collected from the EPM and OF show no differences in behaviour between PWS-cr mice and their wild-type littermates. Our results suggest no obvious indications of anxiety phenotype in the PWS-cr mouse model.

\section{Locomotor activity}

There was no effect of genotype on the total number of beam breaks made in the LMA test over the span of the two sessions $\left(X^{2}(2)=4.625\right.$, $p=0.099$ ), or on a total number of runs made from one end of the chamber to the other $\left(x^{2}{ }_{(2)}=1.237, p=0.539\right)$. Further analysis of the data in $30 \mathrm{~min}$ time bins showed that all animals exhibited habituation to the environment within and between sessions, as indicated by the steady decrease of a number of runs (Fig. 1C; $X^{2}(3)=$ $347.75, p<0.001 ; X^{2}(1)=49.953, p<0.001$ ) and beam breaks (Fig. 1D; $\left.X^{2} \quad(3)=197.83, p<0.001 ; X^{2} \quad(1)=33.562, p<0.001\right)$ over time. However, there was no statistically significant difference in habituation behaviour between the two genotypes $\left(x^{2}(2)=3.342, p=0.342\right.$; $\left.X^{2}{ }_{(2)}=1.761, p=0.222\right)$. Overall, the results from this test showed no locomotor activity phenotype in the PWS-cr mice. 

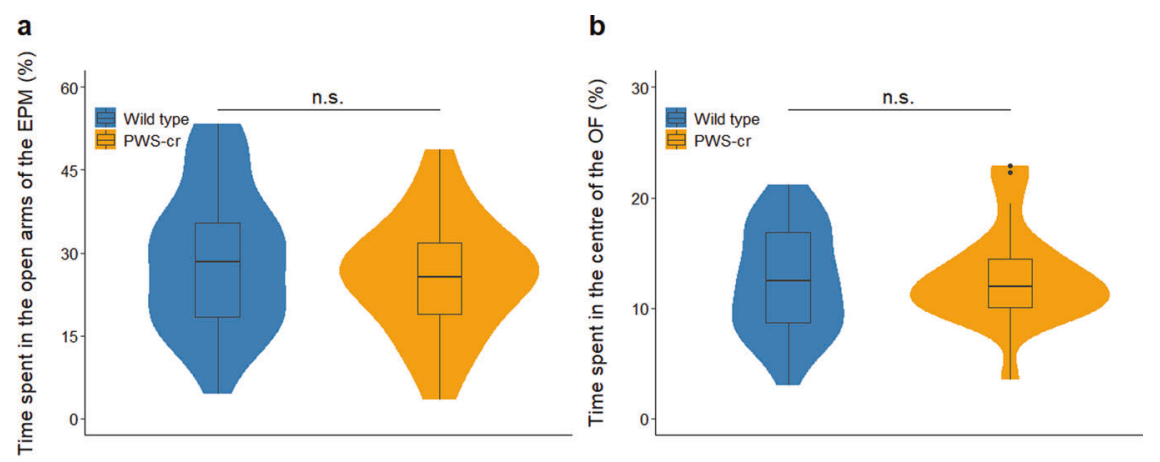

c

d
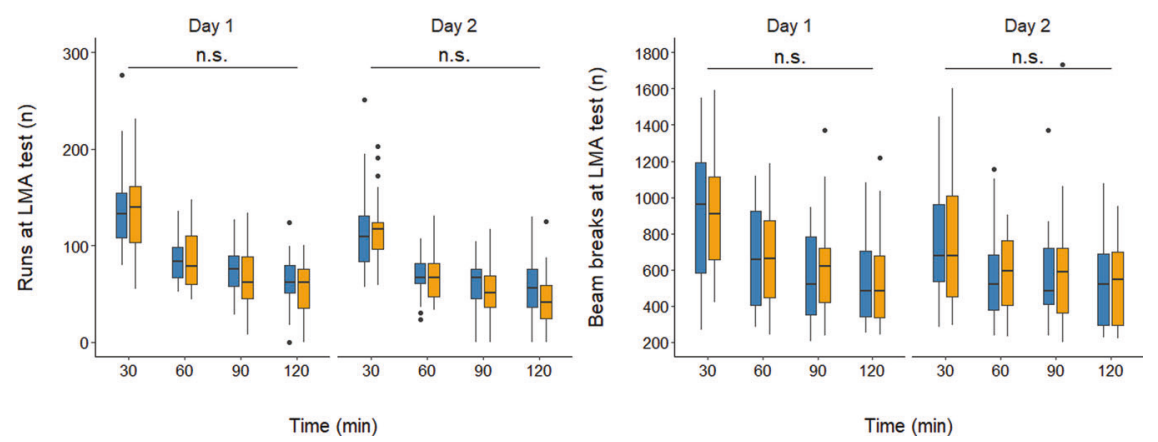

Fig. 1 Behaviours recorded at the elevated plus maze, open field arena and locomotor activity test. The elevated plus-maze task (a) showed no effect of genotype on the percentage of time spent in the open arms of the maze (PWS-cr $=23, W T=31$ ). Similarly, data from the open field test (b) showed no difference in the percentage of time spent in the centre square of the arena between PWS-cr mice $(n=23)$ and their wild-type littermates $(n=31)$. Locomotor activity was tested in custom-made chambers with fitted infrared beams, which tracked the movement of the animals in two-hour sessions on two consecutive days. The number of beam breaks (d) and the number of runs from one end of the chamber to the other (c) were considered the main measures of activity. The test showed decreased locomotor activity between and within sessions $(p<0.001)$, which is indicative of habituation to the environment. There was no effect of genotype on the behaviour examined by this test (PWS-cr $=23, \mathrm{WT}=31$ ).

\section{Acoustic startle response and pre-pulse inhibition}

Acoustic startle response (ASR) and pre-pulse inhibition (PPI) to acoustic startle response were measured at pulse intensities of $120 \mathrm{~dB}$ and $105 \mathrm{~dB}$. A total of thirteen ASR-alone pulses were presented to the mice at the two intensities, and both induced a significantly reduced startle response in the PWS-cr mice (Fig. 2A; $X{ }^{2}{ }_{(2)}=6.5292, p=0.03821$; Fig. $\left.2 \mathrm{~B} ; X^{2}{ }_{(2)}=14.562, p<0.001\right)$. Prepulses of $8 \mathrm{~dB}$ and $16 \mathrm{~dB}$ played $100 \mathrm{~ms}$ prior to the $120 \mathrm{~dB}$ and $105 \mathrm{~dB}$ pulses inhibited the startle response up to a mean average of $74.09 \%( \pm 1.84 \%$ SEM) and $56.1 \%( \pm 4.86 \%$ SEM) respectively, indicating operational sensory-motor gating in both groups of mice (Figs. 2C, D). Genotype had no effect on pre-pulse inhibition at either pulse intensity $\left(X 2_{(2)}=5.376, p=0.251 ; X^{2}{ }_{(2)}=2.556\right.$, $p=0.635)$. Overall, the results from the ASR and PPI tests show no sensory-motor gating phenotypes in the PWS-cr mouse model, but a reduced startle response to acoustic stimuli.

Following the $120 \mathrm{~dB}$ and $105 \mathrm{~dB}$ ASR and PPI schedules, mice were exposed to a gradually increasing ASR-alone pulse intensity, ranging from $70 \mathrm{~dB}$ to $120 \mathrm{~dB}$. As expected, the increasing intensity resulted in increasing response $\left(X^{2}{ }_{(12)}=253.28, p<\right.$ 0.001). These data suggest that the difference in ASR between PWS-cr and wild-type mice emerges at $>100 \mathrm{~dB}$ intensity (Supplementary Figure 1). However, there was no effect of genotype $\left(x_{(12)}^{2}=20.49, p=0.058\right)$ and no interaction between genotype and pulse intensity $\left(X^{2}(10)=9.27, p=0.507\right)$.

\section{5-choice serial reaction time task}

Behaviours relating to visuo-spatial attention and control response were investigated in the 5-CSRTT, where mice were trained to respond correctly to stimuli in order to receive condensed milk dilution as a reward. Prior examination showed an increased preference towards condensed milk dilution over water in all animals $\left(X^{2}{ }_{(3)}=68.461, p<0.001\right)$, thus validating its use as a motivational reward. There was no significant distinction between the preference to the reward between PWS-cr and wild type (Fig. $3 \mathrm{~A}^{\prime} \mathrm{X}^{2}{ }_{(2)}=0.5827$, $p=0.7473)$ and thus no indications of increased interest in the PWScr mice towards the reward, which was an important consideration in the interpretation of the results from the operant tasks.

The average number of sessions required to reach baseline performance in the 5-CSRTT did not differ between PWS-cr (mean 98.6, $\pm 7.3 \mathrm{SEM}$ ) and wild-type (mean 90.8, $\pm 5.7 \mathrm{SEM}$ ) mice $\left(F_{3,40}=0.703, p=0.802\right)$. Performance at baseline conditions of the 5-CSRTT showed no effect of genotype on task accuracy (Fig. $\left.3 \mathrm{~B} ; F_{(3,42)}=0.127, p=0.724\right)$, omissions (Fig. $3 C, F_{(3,42)}=0.104$; $p=0.749$ ), premature responses (Fig. $3 D ; F_{(3,40)}=0.515, p=$ 0.361 ) and all other parameters recorded at these conditions of the task.

The conditions of the 5-CSRTT were manipulated in order to tease out subtler aspects of attention and impulsivity. Behaviour was inspected at a stimulus duration of shortened presentation, all introduced in pseudorandom order within one 5-CSRTT session. The shortened stimulus duration task lowered the overall task accuracy $\left(X^{2}{ }_{(3)}=41.114, p<0.001\right)$, and this performance decline was not influenced by genotype (Fig. $4 \mathrm{~A} ; \mathrm{X}^{2}{ }_{(1)}=1.642, p=0.2$ ). There was no effect of genotype on percentage omissions (Fig. 4B; $\mathrm{X}^{2}{ }_{(1)}=0.357, p=0.551$ ) or any of the other parameters that were investigated in the task.

An increase of the inter-trial intervals was introduced in a different session in order to induce premature responses in the task and tease out a potential impulsivity phenotype. The inter- 
a
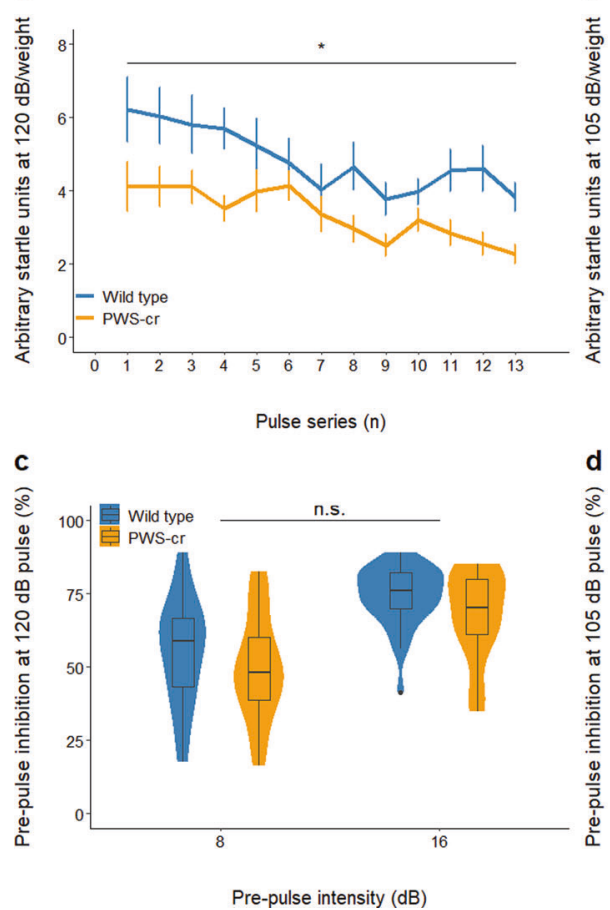

b
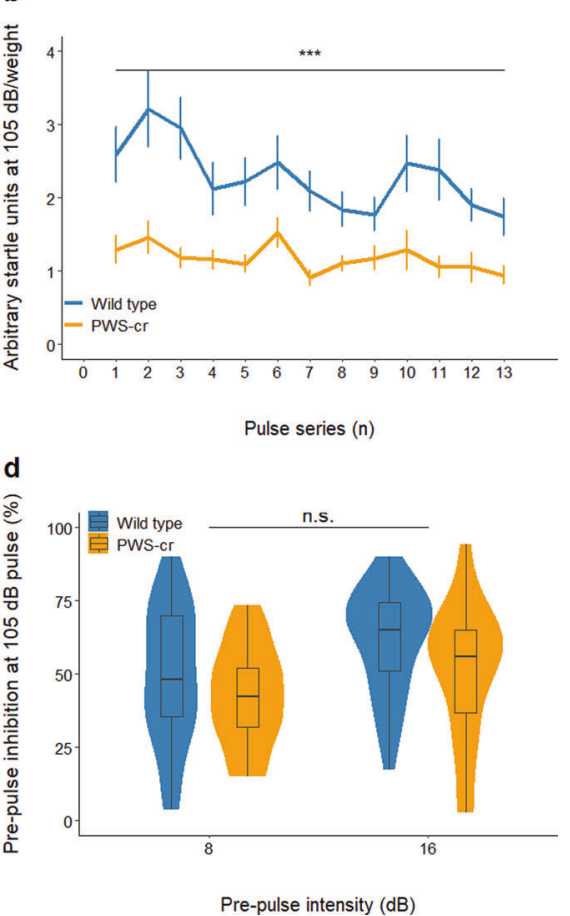

Fig. 2 Acoustic startle and pre-pulse inhibition. The startle response to a series of 13 acoustic stimuli at $120 \mathrm{~dB}$ (a) and $105 \mathrm{~dB}$ (b) was significantly reduced in PWS-cr mice $(n=23)$ compared to their wild-type littermates $(n=31)\left({ }^{*} p<0.05,{ }^{* * *} p<0.001\right)$. Pre-pulse inhibition of the acoustic startle response was measured by presenting noises of $8 \mathrm{~dB}$ and $16 \mathrm{~dB} 70 \mathrm{~ms}$ before the pulses of $120 \mathrm{~dB}$ (c) and 105 (d). The prepulse inhibition data showed operational sensory-motor gating, which was not affected by genotype (PWS-cr $=23, \mathrm{WT}=31$ ).

a

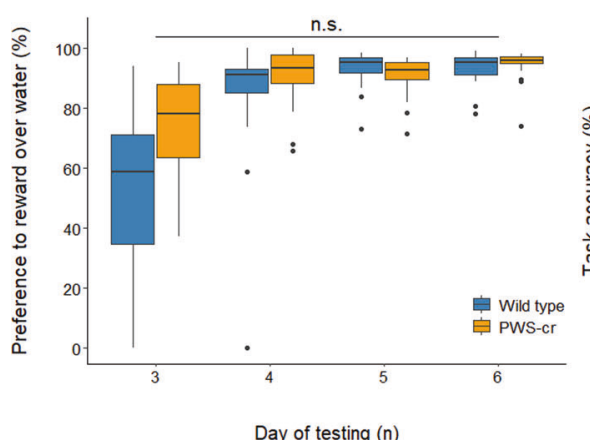

C

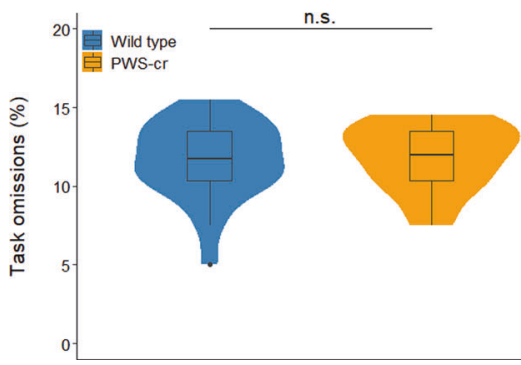

b

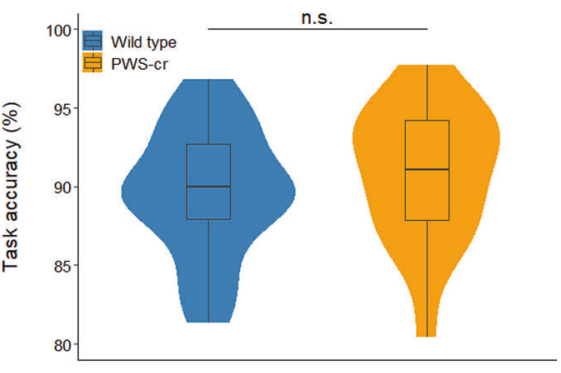

d

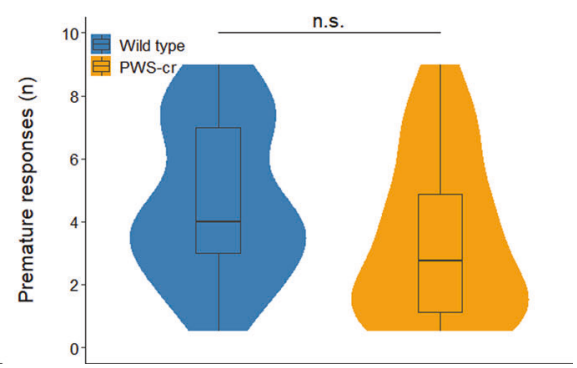

Fig. 3 Condensed milk test and 5-CSRTT performance at baseline conditions. Preference to the condense milk reward over the water was tested over the span of days 3 to 6 from the condense milk test (a). Over the course of these four days the preference for the reward increased significantly $(p<0.001)$ as expected for the purposes of the 5-CSRTT, and this tendency was not affected by genotype (PWS-cr=21, WT $=22$ ). Performance at baseline conditions of the 5-CSRTT also showed no difference between PWS-cr mice $(n=20)$ and their wild-type littermates $(n=23)$ in accuracy of performance (b), percentage omissions (c), and a number of premature responses (d). 
a

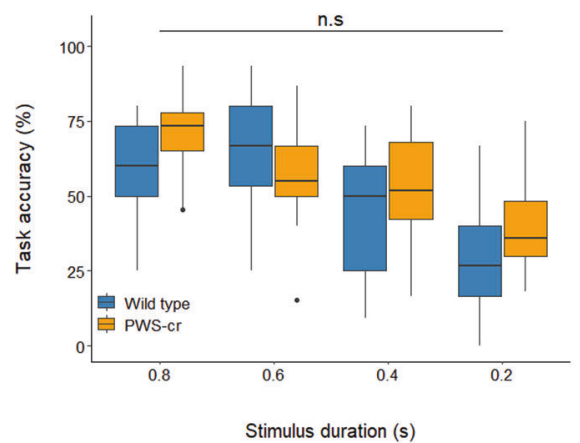

c

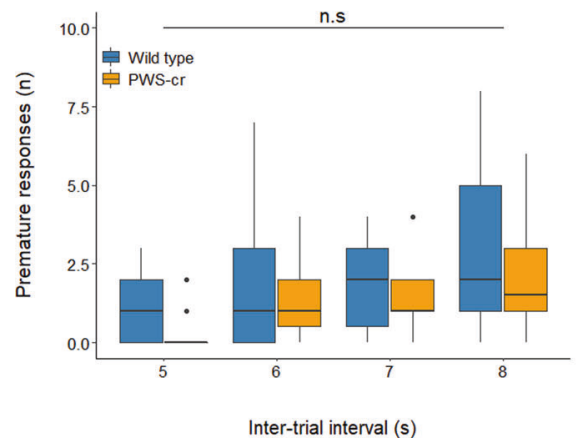

b

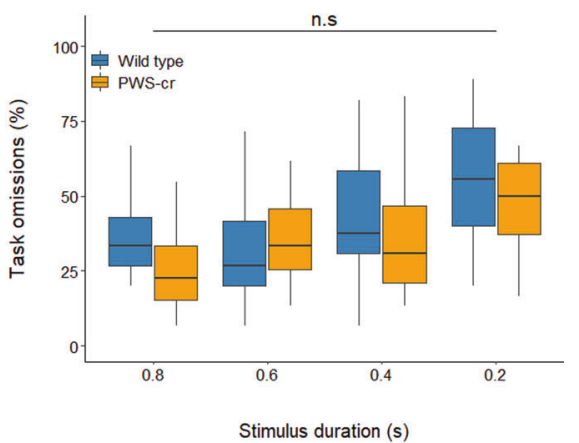

d

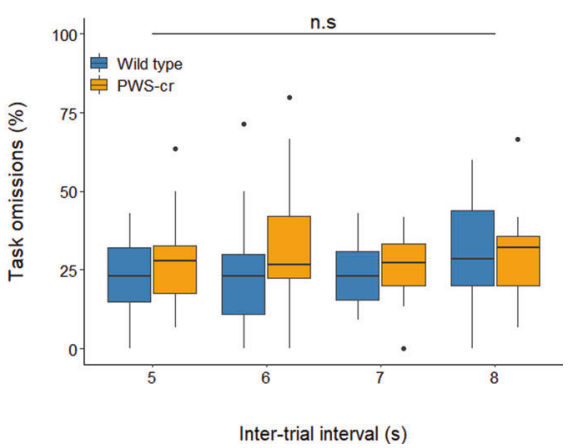

Fig. 4 Shortened stimulus duration and increased inter-trial intervals at the 5-CSRTT. Shortening the stimulus duration at the 5-CSRTT reduced performance accuracy $(p<0.001)$ (a) and increased percentage of trial omissions $(p<0.001)$ (b) but revealed no difference between the behaviour of PWS-cr mice $(n=15)$ and their wild type littermates $(n=21)$. Increasing the inter-trial intervals lead to an increase in premature responses $(p<0.001)$, which was not affected by genotype (PWS-cr=18, WT $=24)$ (c). Omissions were also not affected by genotype in this task manipulation (d).

Table 1. Summary table comparing behavioural and cognitive findings in the PWS-IC and PWS-cr mouse model for Prader-Willi syndrome.

Behavioural domain and measures

Anxiety

Activity

Sensory motor gating

Attention (5-CSRTT)

Impulse control (5-CSRTT, baseline)
Behaviour in PWS model compared to WT littermates PWS-IC [17, 18]

$\equiv$

$\equiv$

$\downarrow$

$\uparrow$

$\downarrow$

$\equiv$
PWS-cr

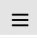

$\equiv$

$\equiv$

$\downarrow$

The PWS-IC mice exhibit a range of behavioural phenotypes of relevance to psychiatric illness, in particular increased startle response and decreased PPI, and reduced attention $[17,18]$. Testing the PWS-cr mice in equivalent conditions (same genetic background, same behavioural equipment, testing rooms, and protocols) revealed no differences, apart from a reduction in acoustic startle response.

trial interval increase leads to a rise in the number of premature responses as predicted $\left(X^{2}{ }_{(3)}=32.131, p<0.001\right)$, but this tendency was not affected by genotype (Fig. $4 C ; X^{2}{ }_{(2)}=4.966$, $p=0.084)$. The percentage of omissions at this task was also not significantly different between PWS-cr mice and their wild-type littermates (Fig. 4D; $X^{2}{ }_{(2)}=0.015, p=0.992$ ), and neither were any of the other examined parameters. Overall, the data collected from the 5-CSRTT showed no indications of an attention or impulsivity phenotype in the PWS-cr mouse model.

RNA-sequencing of whole brain neonatal tissue

The results from our behavioural study demonstrated a vastly different behavioural and cognitive profile in the PWS-cr mice compared to the previously examined PWS-IC mouse (Table 1). In order to investigate the gene expression changes underlying these behavioural differences, we conducted an RNA-sequencing assay on whole-brain tissue from both mouse models in parallel. While all behavioural tasks were conducted on adult individuals, for the RNA-sequencing study we took tissue from neonatal mice, as a genetic risk for psychiatric illness has been shown to manifest from early developmental stages $[48,49]$. Furthermore, qPCR assay (see Supplementary Table 1) of PWS genes in wild-type and PWSIC mouse whole brain tissue at three developmental stages (E13.5, E18.5, P0), showed that Snord115, Snord116, Necdin had significantly reduced expression in the PWS-IC samples, while Ube3a was significantly overexpressed (Supplementary figure $2 ; t(6)=11.788$, 
a

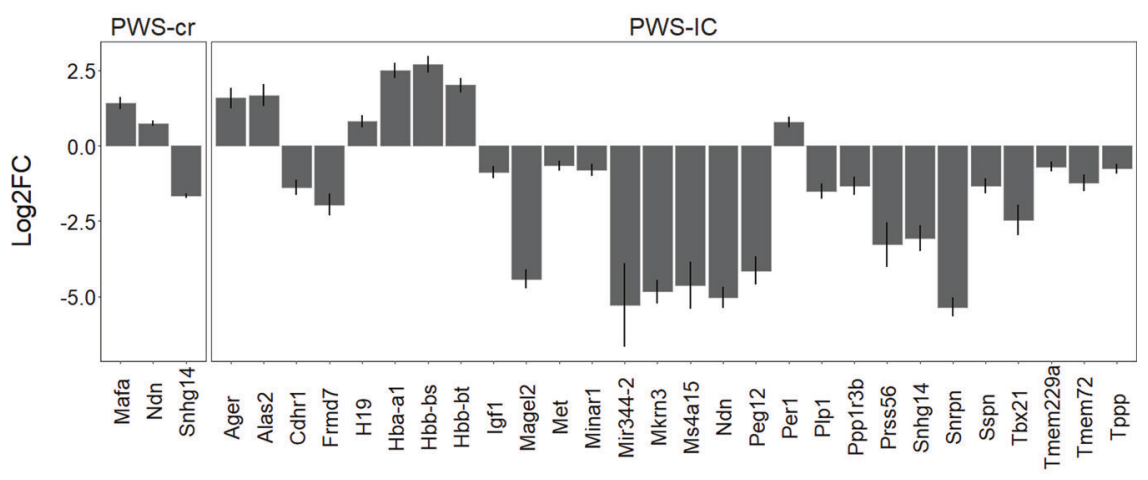

b

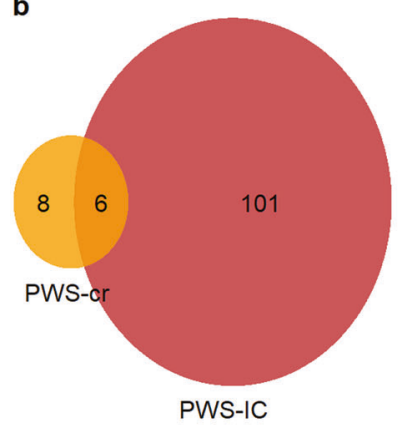

C

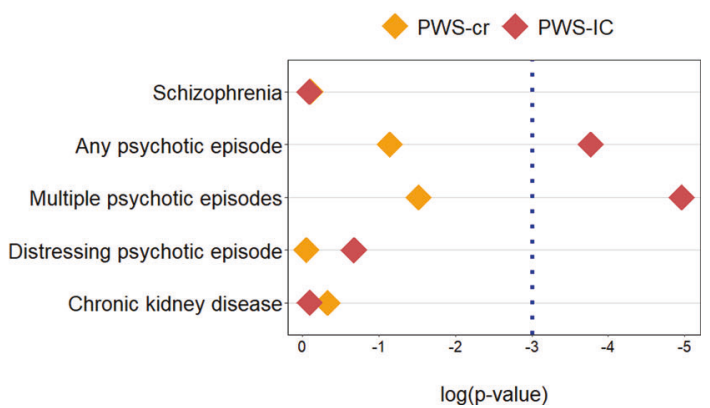

Fig. 5 RNA-sequencing assay. Differential gene expression analysis (a) showed overexpression of Necdin and Mafa in the PWS-cr tissue, as well as an expected loss of expression of the long non-coding RNA Snhg14 that acts as a host for the critical interval. Among the differentially expressed genes in the PWS-IC tissue were the genes from the PWS locus, and notably the circadian clock regulator Per1 and the insulin growth factor lgf1. Predicted genes of unknown function were excluded from the graph. The y axis on this graph (a) shows log2 fold change of expression, error bars indicate standard error of the mean. Differentially expressed genes and isoforms were pooled for enrichment analyses; PWS-cr and PWS-IC pooled genes had 6 genes in common (b). Gene set analysis of pooled differentially expressed genes and isoforms (c) showed significant enrichment of common genetic variants associated with the experience of psychotic episodes, but not schizophrenia. GWAS summary data from a chronic kidney disease study was used as a negative control.

$p<0.001 ; t(6)=-6.415, p<0.001 ; t(6)=-24.033, p<0.001 ; t(6)=$ $-12.956, p<0.001)$, as well as at stage PO $(t(8)=-3.573$, $p=0.007 ; t(8)=-6.297, p<0.001 ; t(8)=-7.4468, p<0.001 ; t(8)$ $=-6.415, p=0.033)$.

PWS-cr differentially expressed genes and isoforms. Visualization of the aligned RNA-sequencing reads in the UCSC browser confirmed that the deletion in the PWS-cr mouse model mode spans all copies of Snord116 snoRNA and five of the six Ipw exons (Supplementary Figure 3). However, differential gene expression analysis showed only very subtle differences between the PWS-cr mice and their wild-type littermates after Benjamini-Hochberg adjustment for multiple testing. In total, there were seven differentially expressed genes (DEGs) in brain tissue from the PWS-cr mouse model (Fig. 5a and Appendix 3). Among them was the Snhg14 non-coding RNA, which acts as a host for the critical interval $\left(z=21.846, p_{\text {adj }}<0.001\right)$. The Mafa gene encoding a transcription factor that regulates pancreatic beta cell-specific expression of the insulin gene was upregulated in the absence of the critical interval $\left(z=-7.014, p_{\text {adj }}<0.001\right)$. Notably, the Necdin growth suppressor which is one of the imprinted genes of the PWS locus, was upregulated in the PWS-cr mouse brain tissue $\left(z=-8.621, p_{\text {adj }}<0.001\right)$. The remaining four DEGs were predicted genes of unknown function, two of which (Gm44559 and Gm44562) fall within intron $4 / 12$ of the Ube3A, and Gm44831 which falls into $I p w$ in the critical interval.

In addition to differential gene expression, the read depth used in our RNA-seq analysis allowed analysis of isoform switches with functional consequences in order to investigate whether the genes in the critical interval play a regulative role in alternate splicing. Seven genes showed significantly different patterns of splicing in the PWS-cr mouse brain (Appendix 4), and there was no overlap between them and the DEGs. Among these was dualspecificity tyrosine-phosphorylation-regulated kinase (Dyrk3), which plays a role in the dissolution of stress granules and in the early secretory pathway [50]. The DEGs and isoforms were pooled together for a gene ontology analysis, which revealed no enrichments.

PWS-IC differentially expressed genes and isoforms. In contrast to PWS-Cr, analysis of PWS-IC mouse brain tissue RNA-sequencing data primarily clustered by genotype and revealed 59 significantly DEGs compared to their wild-type littermates (Fig. 5a; Appendix 5). This included Snhg14, the non-coding RNA that host the Snord116 and Ipw, but also, as expected, the other PWS genes such as Snrpn, Necdin, Mkrn3, and Magel2. Outside the PWS cluster there were other notable DEGs, including the circadian clock regulator Per1 $\left(z=-4.25, p_{\text {adj }}=0.012\right)$, Plp1 $\left(z=6.11, p_{\text {adj }}<0.001\right)$ and insulin growth factor Igf1 $\left(z=4.164, p_{\mathrm{adj}}=0.015\right)$.

Analysis of isoform switches identified 48 differentially spliced genes, and there was no overlap with the differentially expressed genes (Appendix 4). Differentially expressed genes and isoforms were pooled together for a gene ontology term analysis, which were enriched for molecular functions, cellular compartment, and biological compartments relevant to oxygen transport (Appendix 6).

Comparing the combined DEG and isoform data for the two models directly shows that there were a greater number of differences in the PWS-IC mice overall (Fig. 5b). As expected, a good proportion ( 50\%) of the changes seen in PWS-cr were 
shared with those found in PWS-IC (Fig. 5b). However, this overlap was limited to DEGs, as there were no common differentially spliced genes in the PWS-cr and PWS-IC samples.

Enrichment of common genetic variants. We pooled the differentially expressed and differentially spliced gene sets $\left(p_{\text {adj }}<0.05\right)$ together for each mouse model in order to look for enrichment of common genetic variants associated with schizophrenia or psychotic episodes. The analysis was performed using MAGMA's regression gene-set computation with the datasets from genomewide association studies (GWAS) that utilize the UK Biobank, ClozUK, and CKDgen consortium databases respectively [45-47]. The results showed no enrichment of genetic variants of interest in the PWS-cr samples (Fig. 5c). In contrast, results from the PWS-IC model indicated an enrichment of SNPs common for the experience of 'any' and 'multiple' psychotic episodes (Appendix 7; $\beta=0.215, p=0.023, \beta=0.271, p=0.007$ ), but interestingly not for schizophrenia (Fig. $5 c ; \beta=-0.206, p=0.913$ ). As expected, neither gene set was enriched for the negative control dataset, common genetic variants associated with chronic kidney disease $(\beta=-0.161, p=0.909)$.

\section{DISCUSSION}

Our aim in this study was to investigate the differential contribution of genes in the Prader-Willi syndrome imprinted interval to psychiatric illness using mouse models. We have previously demonstrated abnormalities in a number of behavioural and cognitive endophenotypes of relevance to psychiatric illness in a PWS imprinting centre deletion (PWS-IC) mouse model. Here we show that these same behavioural and cognitive measures are not altered in a mouse model for PWS that has a deletion limited to the PWS critical interval (PWS-Cr mice). This suggests that the loss of expression of the PWS-Cr genes does not contribute to the behaviours previously observed in PWS-IC mice. We then used RNA-seq to examine the molecular bases of these differences in behaviour between the PWS-IC and PWS-cr models. Although a number of interesting gene expression and alternate splicing events were detected in the brains of PWS-cr mice, a greater number were seen in the PWS-IC model. Moreover, these changes in gene expression and splicing in the PWS-IC brain were enriched for common genetic variants associated with the experience of psychotic episodes, but not those associated with schizophrenia. These findings shed light on the nature of psychotic illness in PWS and have implications for possible therapeutic strategies.

The behaviour of mice carrying a deletion of the PWS-cr has been extensively characterized with respect to various aspects of the core PWS phenotype including hunger and sleep [23, 51-53], but behaviours relating to psychiatric illness have not been directly studied. Our previous behavioural studies of the PWS-IC model indicated reduced activity, abnormal sensory-motor gating, and deficits in attention [20]. In contrast, here our studies of the PWS-cr mouse model suggests that deletion of the critical region has only very subtle effects on these same behavioural measures. Specifically, the PWS-cr mice showed no abnormalities in anxiety as measured by the EPM and OF tests, locomotor activity, sensorymotor gating as measured by PPI, or attention and impulsivity as measured by the 5-CSRTT. While we cannot rule out Type II error, we note that the PWS-cr behavioural cohort was considerably larger than that used for the PWS-IC mice. Critically the behavioural analysis of PWS-cr mice was conducted in an identical manner to that previously used for the PWS-IC model, including the equipment, procedures, software, location (both Institute and testing rooms), and genetic background strain ( $\mathrm{F} 1$ cross between $\mathrm{C} 57 \mathrm{BI} / 6$ and $\mathrm{CD} 1)$. Therefore, we are confident the comparison between these behavioural studies is robust and valid.

Of the behaviours assessed only ASR was altered in PWS-cr mice. However, in contrast to the enhanced startle response seen in PWS-IC mice, PWS-cr exhibited a decreased startle response. A reduced acoustic startle response could potentially be confounded by hearing loss, and this cannot be completely ruled out here. However, this explanation would seem unlikely given the sensitivity of the response in PWS-cr mice to different startle pulse intensities (Supplementary figure 1), and that there are no records of hearing loss in any mouse models for PWS, or indeed in PWS patients. The combined phenotype of increased startle response and deficient pre-pulse inhibition seen in the PWS-IC mouse model is a long-established endophenotype seen in psychotic illness [54-56] that has been repeatedly shown to have translational utility [31]. Consequently, the lack of any PPI deficit in the PWS-cr deletion model suggests that their reduced startle response is a distinct behavioural outcome. Reduced startle response has been reported in individuals with major depressive disorder and anhedonia, as well as an effect of anxiolytic drugs [57-59]. With this in mind, the reduced ASR seen in PWS-cr mice could fit the psychiatric profile of individuals with PWS that carry deletions spanning the critical interval, who are prone to depression and anxiety, but not psychosis [9-11]. However, a broader variety of behavioural analysis is required to properly establish a depression phenotype in the PWS-cr mice.

The behavioural comparison of these two models indicates that the PWS-cr mice do not exhibit the same range of cognitive and psychotic illness endophenotypes as seen in the PWS-IC mice (Table 1). We next explored the molecular bases for these behavioural differences using RNA-seq. Analysis of RNA-seq data comparing PWS-cr whole brain with wild-type littermates revealed only very subtle changes of gene expression and alternative splicing, with seven differentially expressed genes (four predicted transcripts) and five differentially spliced transcripts. This may seem at odds with previous findings demonstrating differential expression of upwards of 200 genes in PWS-cr mice [27, 60, 61]. However, these studies looked at gene expression in more focused tissues (e.g. hypothalamus) or cell types, whereas our study examined bulk whole brain, therefore obscuring some of the subtler regions- or cell-specific changes and revealing only the strongest effects across the whole brain. In addition to Snord116 and $I p w$, which are directly affected by the mutation (Supplementary figure 2), our data suggest that the PWS-cr deletion also leads to misexpression of the PWS interval gene Necdin, which is an important regulator of neuronal outgrowth and differentiation $[62,63]$, loss of which is associated with motor deficits and enhanced learning and memory [64, 65]. Whether the increased expression of Necdin is due to loss of suppression by Snord116 and/or I $w$, in a manner similar to the regulatory loop between Snord116 and Snord115 [60] or the suppressive role of IPW on the maternally expressed genes from the DLK1-DIO3 imprinted cluster [61], remains to be established. Nevertheless, it could be that this altered expression contributes to the learning and memory deficits seen in PWS-cr mice [66].

In contrast to the limited changes seen in the PWS-cr model, RNA-seq analysis of the PWS-IC whole brain revealed a markedly larger number of DEGs and isoform changes. We suggest these gene expression changes may contribute to behavioural and cognitive deficits seen in the PWS-IC but not the PWS-cr mice and, by extension, may underpin the increased incidence of psychotic illness seen in individuals with imprinting centre deletions or mUPD [13]. In line with this, as well as altered expression of a greater number of genes from within the PWS interval, there were changes in a number of other neurally important genes. These included reduced expression of the myelin protein proteolipid protein Plp1 which has been linked to spatial attention [67]. There was also a significant increase in expression of the circadian rhythm gene, Per1 [68] in the PWS-IC brain. Critically, all our samples were collected within a limited time window in the morning, suggesting that any change in expression may be due to loss of expression of several PWS genes with known circadian rhythm effects $[52,69]$. Of most direct relevance to psychiatric 


\section{Genetic liability for schizophrenia}

\section{Genetic liability for psychosis}

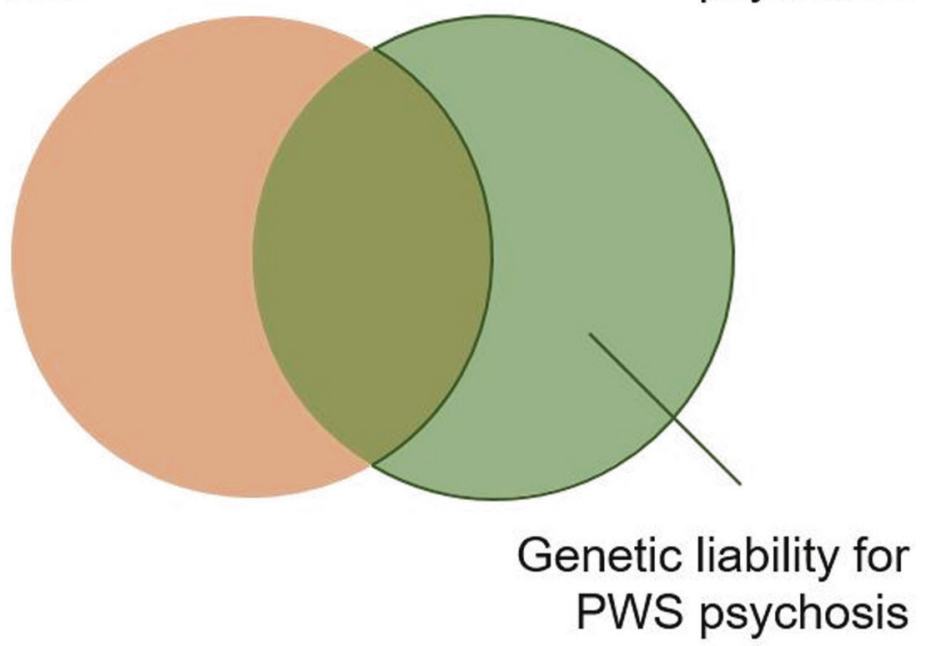

Fig. 6 Model of genetic liability for psychosis in individuals with PWS. Gene set analysis of differentially expressed genes and differentially used isoforms in whole neonatal mouse brain showed enrichment of genes relevant to psychotic experiences, but not schizophrenia. Although schizophrenia and psychotic experiences have a shared genetic liability, they are ultimately distinct psychiatric conditions phenotypically and genetically. We propose that the genetic liability for psychosis in PWS is distinct from that of schizophrenia.

illness were the changes to the gamma-aminobutyric acid (GABA) A receptor subunit gamma 3 gene Gabrg3, which exhibited increased usage of a truncated isoform lacking the neurotransmitter-gated ion-channel ligand-binding domain (NGIC-LBD) and neurotransmitter-gated ion-channel transmembrane region (NGIC-TMR). The GABA receptors play a key role in the function of the central nervous system and this alternate isoform usage could have an effect on various phenotypes including psychotic illness [70, 71]. This further ties in with a study by Webb et al. [72], which showed that in a significant proportion of the PWS-del individuals that exhibit psychosis, carry a larger deletion that spans part of GABRG3. This suggests that the GABA receptor could also be contributing to psychotic illness in individuals with the mUPD15 genotype due to reduced functionality caused by differential isoform usage.

In order to more formally assess the overall relevance of these transcriptomic changes to psychiatric illness, we examined the DEGs and isoforms for the enrichment of genetic variants relevant to psychosis. The GWAS data we used to look for a genetic correlation between our samples with the occurrence of psychotic episodes was from a recent UK Biobank study that sampled individuals with a history of psychotic experiences including hallucinations and delusional ideation, but specifically without a diagnosis of schizophrenia [47]. The authors found a shared genetic liability with schizophrenia from an external GWAS dataset [46], which we also used for our analysis here. Unsurprisingly given the limited number of changes, there was no enrichment in the gene expression changes seen in the PWS-cr neonate brain. However, there was an enrichment of genetic variants common to the experience of psychotic episodes in the gene expression changes seen in the PWS-IC neonate brain. Furthermore, among the genes that carried common SNPs of psychotic experiences were the paternally expressed PWS genes Magel2, Necdin, and Mkrn3. The findings substantiate the relevance of the gene expression changes and behavioural endophenotype observed in the PWS-IC mouse model to the psychiatric profile of individuals with an IC deletion or an mUPD of chromosome 15, but also suggest a contribution of some of the PWS PEGs to these phenotypes. Strikingly, there was not an enrichment of genetic variants associated with schizophrenia in the gene set analysis, which suggests distinct genetic liability of psychosis in PWS from schizophrenia (Fig. 6). This finding provides a biological signal that confirms the observation that the psychotic illness seen in PWS is distinct from schizophrenia [12] and should not be treated in the same manner [17].

Overall, our findings suggest a cumulative effect of several of the genes from the PWS locus on the behavioural endophenotypes typical of Prader-Willi syndrome. The critical interval deletion, albeit important in the core phenotypes seen in PWS models, only induced very subtle behavioural and cognitive differences in the studies we conducted. By comparing the gene expression and isoform profile of the PWS-Cr and PWS-IC lines were able to identify brain molecular changes of relevance to the psychiatric profile of PWS, underlined by the enrichment of genetic variants for psychotic episodes in the PWS-IC transcriptomic changes. The fact that genetic variants for schizophrenia showed no enrichment reflects the distinct nature of psychotic illness in PWS and could have implications for therapeutic strategies used to treat psychosis in individuals with PWS.

\section{DATA AVAILABILITY}

All behavioural data (Appendices 1 and 2) and all RNA-seq data, including gene ontology and genetic enrichment data (Appendices 3-7) are publicly available at the following 'Open science framework' link: https://osf.io/wnx8r/.

\section{REFERENCES}

1. McNamara Gl, Isles AR. Dosage-sensitivity of imprinted genes expressed in the brain: 15q11-q13 and neuropsychiatric illness. Biochem Soc Trans. 2013;41:721-6.

2. Cassidy SB, Schwartz S, Miller JL, Driscoll DJ. Prader-Willi syndrome. Genet Med. 2011;14:10-26.

3. Angulo MA, Butler MG, Cataletto ME. Prader-Willi syndrome: a review of clinical, genetic, and endocrine findings. J Endocrinological Investig. 2015;38:1249-63.

4. Whittington J, Holland A. A review of psychiatric conceptions of mental and behavioural disorders in Prader-Willi syndrome. Neurosci Biobehav Rev. 2018;95:396-405.

5. Thuilleaux D, Laurier V, Copet P, Tricot J, Demeer G, Mourre F, et al. A model to characterize psychopathological features in adults with Prader-Willi syndrome. Am J Med Genet A. 2018;176:41-7.

6. Reddy LA, Pfeiffer SI. Behavioral and emotional symptoms of children and adolescents with Prader-Willi Syndrome. J Autism Dev Disord. 2007;37:830-9.

7. Sinnema $M$, Boer $H$, Collin $P$, Maaskant MA, van Roozendaal KE, SchranderStumpel $C T$, et al. Psychiatric illness in a cohort of adults with Prader-Willi syndrome. Res Dev Disabil. 2011;32:1729-35. 
8. Vogels A, De Hert M, Descheemaeker MJ, Govers V, Devriendt K, Legius E, et al. Psychotic disorders in Prader-Willi syndrome. Am J Med Genet A. 2004;127A:238-43.

9. Sahoo T, del Gaudio D, German JR, Shinawi M, Peters SU, Person RE, et al. PraderWilli phenotype caused by paternal deficiency for the HBII-85 C/D box small nucleolar RNA cluster. Nat Genet. 2008;40:719-21.

10. Fontana P, Grasso M, Acquaviva F, Gennaro E, Galli ML, Falco M, et al. SNORD116 deletions cause Prader-Willi syndrome with a mild phenotype and macrocephaly. Clin Genet. 2017;92:440-3.

11. Bieth E, Eddiry S, Gaston V, Lorenzini F, Buffet A, Conte Auriol F, et al. Highly restricted deletion of the SNORD116 region is implicated in Prader-Willi Syndrome. Eur J Hum Genet. 2015;23:252-5.

12. Soni $\mathrm{S}$, Whittington J, Holland AJ, Webb T, Maina EN, Boer $\mathrm{H}$, et al. The phenomenology and diagnosis of psychiatric illness in people with Prader-Willi syndrome. Psychol Med. 2008;38:1505-14.

13. Boer H, Holland A, Whittington J, Butler J, Webb T, Clarke D. Psychotic illness in people with Prader Willi syndrome due to chromosome 15 maternal uniparental disomy. Lancet. 2002;359:135-6.

14. Chamberlain SJ, Brannan $\mathrm{Cl}$. The Prader-Willi syndrome imprinting center activates the paternally expressed murine Ube3a antisense transcript but represses paternal Ube3a. Genomics. 2001;73:316-22.

15. Vogels A, Matthijs G, Legius E, Devriendt K, Fryns JP. Chromosome 15 maternal uniparental disomy and psychosis in Prader-Willi syndrome. J Med Genet. 2003;40:72-3.

16. Isles AR, Ingason A, Lowther C, Walters J, Gawlick M, Stöber G, et al. Parental origin of interstitial duplications at 15q11.2-q13.3 in schizophrenia and neurodevelopmental disorders. PLoS Genet. 2016;12:e1005993.

17. Soni S, Whittington J, Holland AJ, Webb T, Maina $\mathrm{E}$, Boer $\mathrm{H}$, et al. The course and outcome of psychiatric illness in people with Prader-Willi syndrome: implications for management and treatment. J Intellect Disabil Res. 2007;51:32-42.

18. Salminen I, Read S, Hurd P, Crespi B. Does SNORD116 mediate aspects of psychosis in Prader-Willi syndrome? Evidence from a non-clinical population. Psychiatry Res. 2020;286:112858.

19. Crespi B, Read S, Salminen I, Hurd P. A genetic locus for paranoia. Biol Lett. 2018;14:20170694.

20. Relkovic D, Doe CM, Humby T, Johnstone KA, Resnick JL, Holland AJ, et al. Behavioural and cognitive abnormalities in an imprinting centre deletion mouse model for Prader-Willi syndrome. Eur J Neurosci. 2010;31:156-64.

21. Davies JR, Wilkinson LS, Isles AR, Humby T. Prader-Willi syndrome imprinting centre deletion mice have impaired baseline and 5-HT2CR-mediated response inhibition. Hum Mol Genet. 2019;28:3013-23.

22. Doe CM, Relkovic D, Garfield AS, Dalley JW, Theobald DE, Humby T, et al. Loss of the imprinted snoRNA mbii-52 leads to increased 5 htr2c pre-RNA editing and altered 5HT2CR-mediated behaviour. Hum Mol Genet. 2009;18:2140-8.

23. Ding F, Li HH, Zhang S, Solomon NM, Camper SA, Cohen $P$, et al. SnoRNA Snord116 (Pwcr1/MBII-85) deletion causes growth deficiency and hyperphagia in mice. PLoS ONE. 2008;3:e1709.

24. Skryabin BV, Gubar LV, Seeger B, Pfeiffer J, Handel S, Robeck T, et al. Deletion of the MBII-85 snoRNA gene cluster in mice results in postnatal growth retardation. PLoS Genet. 2007;3:e235.

25. Golding DM, Rees DJ, Davies JR, Relkovic D, Furby HV, Guschina IA, et al. Paradoxical leanness in the imprinting-centre deletion mouse model for Prader-Willi syndrome. J Endocrinol. 2017;232:123-35.

26. Pace M, Falappa M, Freschi A, Balzani E, Berteotti C, Lo Martire V, et al. Loss of Snord116 impacts lateral hypothalamus, sleep, and food-related behaviors. JCl Insight. 2020;5:e137495.

27. Polex-Wolf J, Lam BY, Larder R, Tadross J, Rimmington D, Bosch F, et al. Hypothalamic loss of Snord116 recapitulates the hyperphagia of Prader-Willi syndrome. J Clin Invest. 2018;128:960-9.

28. Davies JR, Humby T, Dwyer DM, Garfield AS, Furby H, Wilkinson LS, et al. Calorie seeking, but not hedonic response, contributes to hyperphagia in a mouse model for Prader-Willi syndrome. Eur J Neurosci. 2015;42:2105-13.

29. Walf AA, Frye CA. The use of the elevated plus maze as an assay of anxietyrelated behavior in rodents. Nat Protoc. 2007;2:322-8.

30. Prut $L$, Belzung $C$. The open field as a paradigm to measure the effects of drugs on anxiety-like behaviors: a review. Eur J Pharmacol. 2003;463:3-33.

31. Powell SB, Weber M, Geyer MA. Genetic models of sensorimotor gating: relevance to neuropsychiatric disorders. Curr Top Behav Neurosci. 2012;12:251-318

32. Robbins T. 5-choice serial reaction time task: behavioural pharmacology and functional neurochemistry. Psychopharmacology. 2002;163:380. 362.

33. Humby T, Laird FM, Davies W, Wilkinson LS. Visuospatial attentional functioning in mice: interactions between cholinergic manipulations and genotype. Eur J Neurosci. 1999;11:2813-23.
34. Humby T, Wilkinson L, Dawson G. Assaying aspects of attention and impulse control in mice using the 5-choice serial reaction time task. Curr Protoc Neurosci. 2005;31:8.5H.1-8.5H.15.

35. Bates $D$, Maechler M, Bolker B, Walker S. Fitting linear mixed-effects models using Ime4. J Stat Softw. 2015;67:1-48.

36. Dobin A, Gingeras TR. Mapping RNA-seq Reads with STAR. Curr Protoc Bioinforma. 2015;51:11.14.11-11.14.19.

37. Liao Y, Smyth G, Shi W. featureCounts: an efficient general purpose program for assigning sequence reads to genomic features. Bioinformatics. 2014;30:923-30.

38. Anders S, Huber W. Differential expression analysis for sequence count data. Genome Biol. 2010;11:R106.

39. Bray NL, Pimentel H, Melsted P, Pachter L. Near-optimal probabilistic RNA-seq quantification. Nat Biotechnol. 2016;34:525-7.

40. Vitting-Seerup K, Sandelin A. The landscape of isoform switches in human cancers. Mol Cancer Res. 2017;15:1206-20.

41. Ritchie ME, Phipson B, Wu D, Hu Y, Law CW, Shi W, et al. limma powers differential expression analyses for RNA-sequencing and microarray studies. Nucleic Acids Res. 2015;43:e47-e47.

42. Anders $S$, Reyes A, Huber W. Detecting differential usage of exons from RNA-seq data. Genome Res. 2012;22:2008-17.

43. Punta M, Coggill PC, Eberhardt RY, Mistry J, Tate J, Boursnell C, et al. The Pfam protein families database. Nucleic Acids Res. 2011;40:D290-D301.

44. Raudvere U, Kolberg L, Kuzmin I, Arak T, Adler P, Peterson H, et al. g:Profiler: a web server for functional enrichment analysis and conversions of gene lists (2019 update). Nucleic Acids Res. 2019;47:W191-8.

45. Wuttke M, Li Y, Li M, Sieber KB, Feitosa MF, Gorski M, et al. A catalog of genetic loci associated with kidney function from analyses of a million individuals. Nat Genet. 2019;51:957-72.

46. Pardiñas AF, Holmans P, Pocklington AJ, Escott-Price V, Ripke S, Carrera N, et al. Common schizophrenia alleles are enriched in mutation-intolerant genes and in regions under strong background selection. Nat Genet. 2018;50:381-9.

47. Legge $S E$, Jones HJ, Kendall KM, Pardiñas AF, Menzies $G$, Bracher-Smith $M$, et al. Association of genetic liability to psychotic experiences with neuropsychotic disorders and traits. JAMA Psychiatry. 2019;76:1256-65.

48. Mistry S, Harrison JR, Smith DJ, Escott-Price V, Zammit S. The use of polygenic risk scores to identify phenotypes associated with genetic risk of schizophrenia: systematic review. Schizophrenia Res. 2018;197:2-8.

49. Schork AJ, Won H, Appadurai V, Nudel R, Gandal M, Delaneau O, et al. A genomewide association study of shared risk across psychiatric disorders implicates gene regulation during fetal neurodevelopment. Nat Neurosci. 2019;22:353-61.

50. Wippich F, Bodenmiller B, Trajkovska MG, Wanka S, Aebersold R, Pelkmans L. Dual specificity kinase DYRK3 couples stress granule condensation/dissolution to mTORC1 signaling. Cell. 2013;152:791-805.

51. Qi Y, Purtell L, Fu M, Lee NJ, Aepler J, Zhang L, et al. Snord116 is critical in the regulation of food intake and body weight. Sci Rep. 2016;6:18614.

52. Lassi G, Priano L, Maggi S, Garcia-Garcia C, Balzani E, El-Assawy N, et al. Deletion of the Snord116/SNORD116 alters sleep in mice and patients with Prader-Willi syndrome. Sleep. 2016;39:637-44.

53. Lassi G, Maggi S, Balzani E, Cosentini I, Garcia-Garcia C, Tucci V. Working-for-food behaviors: a preclinical study in Prader-Willi mutant mice. Genetics. 2016;204:1129-38.

54. Swerdlow NR, Light GA, Sprock J, Calkins ME, Green MF, Greenwood TA, et al. Deficient prepulse inhibition in schizophrenia detected by the multi-site COGS. Schizophr Res. 2014;152:503-12.

55. Geyer MA, Krebs-Thomson K, Braff DL, Swerdlow NR. Pharmacological studies of prepulse inhibition models of sensorimotor gating deficits in schizophrenia: a decade in review. Psychopharmacol. 2001;156:117-54.

56. Geyer MA, Swerdlow NR, Mansbach RS, Braff DL. Startle response models of sensorimotor gating and habituation deficits in schizophrenia. Brain Res Bull. 1990;25:485-98.

57. Kaviani H, Gray JA, Checkley SA, Raven PW, Wilson GD, Kumari V. Affective modulation of the startle response in depression: influence of the severity of depression, anhedonia, and anxiety. J Affect Disord. 2004;83:21-31.

58. Commissaris RL, Fomum EA, Leavell BJ. Effects of buspirone and alprazolam treatment on the startle-potentiated startle response. Depression Anxiety. 2004;19:146-51.

59. Riba J, Rodríguez-Fornells A, Urbano G, Morte A, Antonijoan R, Barbanoj MJ. Differential effects of alprazolam on the baseline and fear-potentiated startle reflex in humans: a dose-response study. Psychopharmacol. 2001;157:358-67.

60. Falaleeva M, Surface J, Shen M, de la Grange P, Stamm S. SNORD116 and SNORD115 change expression of multiple genes and modify each other's activity. Gene. 2015;572:266-73.

61. Stelzer Y, Sagi I, Yanuka O, Eiges R, Benvenisty N. The noncoding RNA IPW regulates the imprinted DLK1-DIO3 locus in an induced pluripotent stem cell model of Prader-Willi syndrome. Nat Genet. 2014;46:551-7. 
62. Lee S, Walker CL, Karten B, Kuny SL, Tennese AA, O'Neill MA, et al. Essential role for the Prader-Willi syndrome protein necdin in axonal outgrowth. Hum $\mathrm{Mol}$ Genet. 2005;14:627-37.

63. Kuwajima T, Nishimura I, Yoshikawa K. Necdin promotes GABAergic neuron differentiation in cooperation with $\mathrm{dlx}$ homeodomain proteins. J Neurosci. 2006;26:5383-92.

64. Muscatelli F, Abrous DN, Massacrier A, Boccaccio I, Le Moal M, Cau P, et al. Disruption of the mouse Necdin gene results in hypothalamic and behavioral alterations reminiscent of the human Prader-Willi syndrome. Hum Mol Genet. 2000;9:3101-10.

65. Andrieu D, Meziane H, Marly F, Angelats C, Fernandez PA, Muscatelli F. Sensory defects in Necdin deficient mice result from a loss of sensory neurons correlated within an increase of developmental programmed cell death. BMC Developmental Biol. 2006;6:56.

66. Adhikari A, Copping NA, Onaga B, Pride MC, Coulson RL, Yang M, et al. Cognitive deficits in the Snord116 deletion mouse model for Prader-Willi syndrome. Neurobiol Learn Mem. 2019;165:106874-106874.

67. Ocklenburg S, Gerding WM, Raane M, Arning L, Genç E, Epplen JT, et al. PLP1 gene variation modulates leftward and rightward functional hemispheric asymmetries. Mol Neurobiol. 2018;55:7691-7700.

68. Albrecht U, Eichele G. The mammalian circadian clock. Curr Opin Genet Dev. 2003;13:271-7.

69. Kozlov SV, Bogenpohl JW, Howell MP, Wevrick R, Panda S, Hogenesch JB, et al. The imprinted gene Magel2 regulates normal circadian output. Nat Genet. 2007;39:1266-72.

70. Taylor SF, Tso IF. GABA abnormalities in schizophrenia: a methodological review of in vivo studies. Schizophr Res. 2015;167:84-90.

71. Ahn K, Gil R, Seibyl J, Sewell RA, D'Souza DC. Probing GABA receptor function in schizophrenia with iomazenil. Neuropsychopharmacology. 2011;36:677-83.

72. Webb T, Maina EN, Soni S, Whittington J, Boer H, Clarke D. et al. In search of the psychosis gene in people with Prader-Willi syndrome. Am J Med Genet A. 2008;146:843-53.

\section{AUTHOR CONTRIBUTIONS}

SKZ and ARI planned the experiments. SKZ performed all behavioural experiments and analyses with input from $\mathrm{TH}$ and ARI; Tissue collection and processing were performed by SKZ and JRD; RNA QC and RNA-seq were performed by JEM, with data analysis carried out by SKZ. SKZ and ARI wrote the paper.

\section{FUNDING}

This work was supported by a UKRI Medical Research Council (MRC) studentship to SZ (1976193), the MRC Centre for Neuropsychiatric Genetics and Genomics (MR/ L010305/1), and UKRI Biotechnology and Biological Sciences Research Council research grant to $\mathrm{ARI}(\mathrm{BB} / \mathrm{J} 016756 / 1)$.

\section{COMPETING INTERESTS}

The authors declare no competing interests.

\section{ADDITIONAL INFORMATION}

Supplementary information The online version contains supplementary material available at https://doi.org/10.1038/s41398-021-01561-x.

Correspondence and requests for materials should be addressed to A.R.I

Reprints and permission information is available at http://www.nature.com/ reprints

Publisher's note Springer Nature remains neutral with regard to jurisdictional claims in published maps and institutional affiliations.

(c) Open Access This article is licensed under a Creative Common Attribution 4.0 International License, which permits use, sharing, adaptation, distribution and reproduction in any medium or format, as long as you give appropriate credit to the original author(s) and the source, provide a link to the Creative Commons license, and indicate if changes were made. The images or other third party material in this article are included in the article's Creative Commons license, unless indicated otherwise in a credit line to the material. If material is not included in the article's Creative Commons license and your intended use is not permitted by statutory regulation or exceeds the permitted use, you will need to obtain permission directly from the copyright holder. To view a copy of this license, visit http://creativecommons. org/licenses/by/4.0/.

(c) The Author(s) 2021 